\title{
Radioterapia Externa Exclusiva en Carcinoma Invasor Avanzado del Cuello del Útero.
}

\section{Exclusive External Radiotherapy in Advanced Invasive Carcinoma of the Cervix.}

*Correspondencia:

Imv524@gmail.com

Teléfono [593] 072614314

Conflicto de intereses: Los autores declaran no tener conflictos de intereses.

Fondos: Ver la página 199

Recibido: 19 Enero 2018

Aceptado: 15 Octubre 2018

Publicado: 30 Diciembre 2018

Membrete bibliográfico:

Morales L, Arciniega E, Bermeo A. Radioterapia Externa Exclusiva en Carcinoma Invasor Avanzado del Cuello del Útero. Rev. Oncol. Ecu 2018;28(3):191-201.

DOI: https://doi.org/10.33821/136

Copyright Morales, et al. Este artículo es distribuido bajo los términos de Creative Commons Attribution License, el cual permite el uso y redistribución citando la fuente y al autor original.

\section{Luis E Morales Vivar ${ }^{1 *}$, Estrellita Arciniega Gutiérrez², Amable Bermeo Flores ${ }^{2}$.}

1. Instituto del Cáncer de Solca Núcleo de Loja, Ecuador.

2. Universidad Nacional de Loja, Ecuador.

\section{Resumen}

Introducción: Una combinación de radioterapia de haz externo y braquiterapia es el estándar de manejo radiante radical para el cáncer cervical avanzado. Algunas pacientes no pueden recibir braquiterapia, por lo que se administran mayores dosis de radioterapia externa exclusiva. Se describen los resultados obtenidos con dicha modalidad terapéutica.

Métodos: En un estudio retrospectivo, descriptivo, entre Julio de 1997 y Agosto del 2005, se evalúa pacientes mayores de 18 años, tratadas en el Instituto del Cáncer de Solca Núcleo de Loja con el diagnóstico inicial de cáncer cervical estadio III B y IVA. Se caracterizan variables: edad, estadio, respuesta al final del tratamiento, tiempo global de tratamiento, sobrevida global, morbilidad tardía y complicaciones. Se presenta los datos usando medidas de resumen de tendencia central y la curva de supervivencia mediante el método Kaplan Meier: Se utilizó el paquete estadístico SPSS 20.0.

Resultados: 25 pacientes con una mediana de edad de 65 años, con cáncer en estadios IIIB y IV recibieron una media de 68 Gy con radioterapia externa exclusiva, en una mediana de 65 días de tiempo global de tratamiento. Hubo respuesta clínica completa en 9 pacientes (36\%); residual tumoral clínico en las 16 restantes (64\%). A los 5 años de seguimiento, 6 pacientes (24\%) se encontraban vivas, sin datos de actividad tumoral locorregional, en las 19 pacientes restantes (76 \%), hubo falla locorregional.

Conclusión: La radioterapia externa exclusiva no ofrece resultados satisfactorios. Debe insistirse en la necesidad de braquiterapia para alcanzar dosis curativas.

Palabras Claves: ENFERMEDADES DEL CUELLO DEL ÚTERO, NEOPLASIAS UTERINAS, RADIOTERAPIA, RADIOTERAPIA AYUVANTE, SUPERVIVENCIA (SALUD PÚBLICA), MORBILIDAD. 
DOI: $10.33821 / 136$

\section{Abstract}

Introduction: A combination of external beam radiotherapy and brachytherapy is the standard therapy for advanced cervical cancer. Some patients can not receive brachytherapy, so are given larger doses of exclusive external radiotherapy. Describes the results obtained with this treatment modality.

Methods: In a retrospective study, between July 1997 and August 2005, evaluating patients treated in Cancer Institute Core SOLCA Loja. Variables is characterized age, stage, end of treatment response, overall treatment time, overall survival, late morbidity. We present data using summary measures of central tendency and the survival curve using the Kaplan Meier method and the statistical program SPSS 20.0 .

Results: 25 patients with a median age of 65 years, cancer stage IIIB and IV received a median of 68 Gy with exclusive external radiotherapy at a median of 65 days overall treatment time. Clinical response was complete in 9 patients (36\%) clinical residual tumor remaining 16 (64\%). At 5 years follow-up, 6 patients (24\%) were alive without locoregional tumor activity data in the remaining 19 patients (76\%), there locoregional failure. Morbidity low and comparable to that reported in the literature.

Conclusion: The exclusive external radiotherapy does not offer satisfactory results. It should be emphasized the need to achieve curative doses with brachytherapy.

Keywords: UTERINE CERVICAL DISEASES, UTERINE NEOPLASMS, RADIOTHERAPY, RADIOTHERAPY, ADJUVANT, SURVIVAL, MORBIDITY.

DOI: $10.33821 / 136$

\section{Introducción}

Según los datos del registro de tumores de Solca-Núcleo de Loja [1], la tasa de incidencia de cáncer de cuello uterino es de 55.3 casos x 100 mil mujeres, constituyéndose como la neoplasia más frecuente en mujeres.

El tamaño del tumor primario es un factor pronóstico [2], la supervivencia y el control local son mejores si el compromiso del parametrio es unilateral en lugar de bilateral [3], en los estudios de modelos de atención de pacientes en estadios IIIA y IIIB, se indica que la supervivencia depende de la extensión de la enfermedad; el compromiso unilateral de la pared pélvica pronostica un mejor desenlace que el compromiso bilateral que, a su vez, pronostica un mejor desenlace que el compromiso del tercio inferior de la pared vaginal.[3] Estos estudios también revelan un aumento progresivo del control local y la supervivencia, paralelo a un aumento progresivo de las dosis paracentrales (punto A) y la administración de tratamiento intracavitario. La tasa más alta de control central se observó con dosis paracentrales (punto A) mayores de $85 \mathrm{~Gy}[4,5]$. Estos tumores en estadio IIIA y B se tratan con Radioterapia y Quimioterapia simultánea, los tumores en estadío IVB se tratan con Radioterapia y Quimioterapia paliativa. 
Habitualmente, por el volumen tumoral, se inicia la terapia radiante administrando externamente las radiaciones ionizantes, para tratamiento de la pelvis cuyo objetivo es irradiar los ganglios regionales y el tumor primario con tejidos vecinos involucrados denominado Teleradioterapia (TRT). Posteriormente, se administran radiaciones intrauterinas en la modalidad de Braquiterapia (BT), que permite alcanzar dosis tumoricidas evitando sobrepasar la tolerancia de los órganos vecinos, principalmente la vejiga y el recto [6-8].

Por condiciones de infraestructura en Solca-Loja, las pacientes que ameriten BT, deben ser referidas a las ciudades de Quito o Guayaquil. Las pacientes que no pueden recibir BT, se les administra incremento de dosis de radiaciones a través de TRT, que, dada la tolerancia de los órganos vecinos, principalmente vejiga, recto y asas intestinales, no permiten alcanzar dosis tumoricidas [9].

En el presente trabajo, se analizan las características clínicas de las pacientes en quiénes se administró TRT, se reporta la supervivencia de pacientes con Ca uterino avanzado en estadíos IIIB y IVA.

\section{Materiales y Métodos}

El presente es un estudio descriptivo, retrospectivo, no experimental, de un solo centro hospitalario, con revisión de las historias clínicas de las pacientes diagnosticadas con carcinoma invasor avanzado del cuello del útero, mayores de edad, tratadas en el área de Radioterapia del Instituto del Cáncer de Solca-Núcleo de Loja, entre Julio de 1997 y Agosto de 2005.

Las pacientes recibieron una primera fase de tratamiento radiante (Teleterapia), usando una Unidad de Cobaltoterapia (energía de 1,25 MeV), mediante la técnica de caja pélvica modificada, adecuada al volumen tumoral macroscópico y al volumen tumoral clínico, en fraccionamiento diario de $200 \mathrm{cGy}$, hasta alcanzar $5.000 \mathrm{cGy}$. Luego, las pacientes que, por volumen o geometría tumoral residual, decisión personal, imposibilidad socioeconómica para desplazarse a los centros de mayor infraestructura, o excesivo diferimiento, no pudieron recibir BT, se les administró 9 sesiones adicionales de TRT, mediante un protocolo de "caja pélvica reducida", al tumor primario y tejidos vecinos, alcanzando $68 \mathrm{~Gy}$.

El universo del estudio lo constituyeron todas las pacientes con los criterios mencionados en quienes se pudieron recopilar todas las variables del estudio. Las variables estudiadas fueron edad, estadio clínico al diagnóstico, tiempo global de tratamiento, respuesta clínica al final de la primera fase de RT externa y luego del incremento de dosis, y sobrevida libre de enfermedad a 5 años, cuantificada desde la fecha de inicio de la terapia hasta la fecha de su último examen de seguimiento, sin recurrencia locorregional o metástasis distantes.

Los términos de respuesta completa o parcial se definieron de acuerdo a los criterios de evaluación de respuesta en tumores sólidos de la OMS. La recurrencia locorregional se definió como la aparición del tumor local o regional (vagina, cérvix, útero, parametrios, pelvis), sola o en conjunto con metástasis distantes. Las complicaciones tardías rectales, 
vesicales e intestinales fueron valoradas de acuerdo al sistema de gradación del RTOG (Radiation Therapy Oncology Group) y el EORTC ( European Organization for Research and Treatment of Cancer) [9].

Para la evaluación de los datos, se usaron medidas de tendencia central como medidas de resumen, frecuencia y porcentajes para variables cuantitativas. Se presenta la información en forma de texto y gráficos, curva Kaplan Meier para la supervivencia global y de acuerdo a la respuesta clínica al final de la terapia radiante. El paquete estadístico Utilizado fue SPPS 20.0 para PC.

\section{Resultados}

Se obtuvieron las historias clínicas de 25 pacientes sometidas al manejo de TRT exclusiva. La mediana de edad de las pacientes del estudio fue de 65 años, con valores extremos de 30 y 89 años de edad. Se encontraron 20 pacientes en estadio clínico IIIB y 5 pacientes en estadio clínico IV (Figura 1).

Figura 1. Grupo de estudio representados por Edad y estadío.

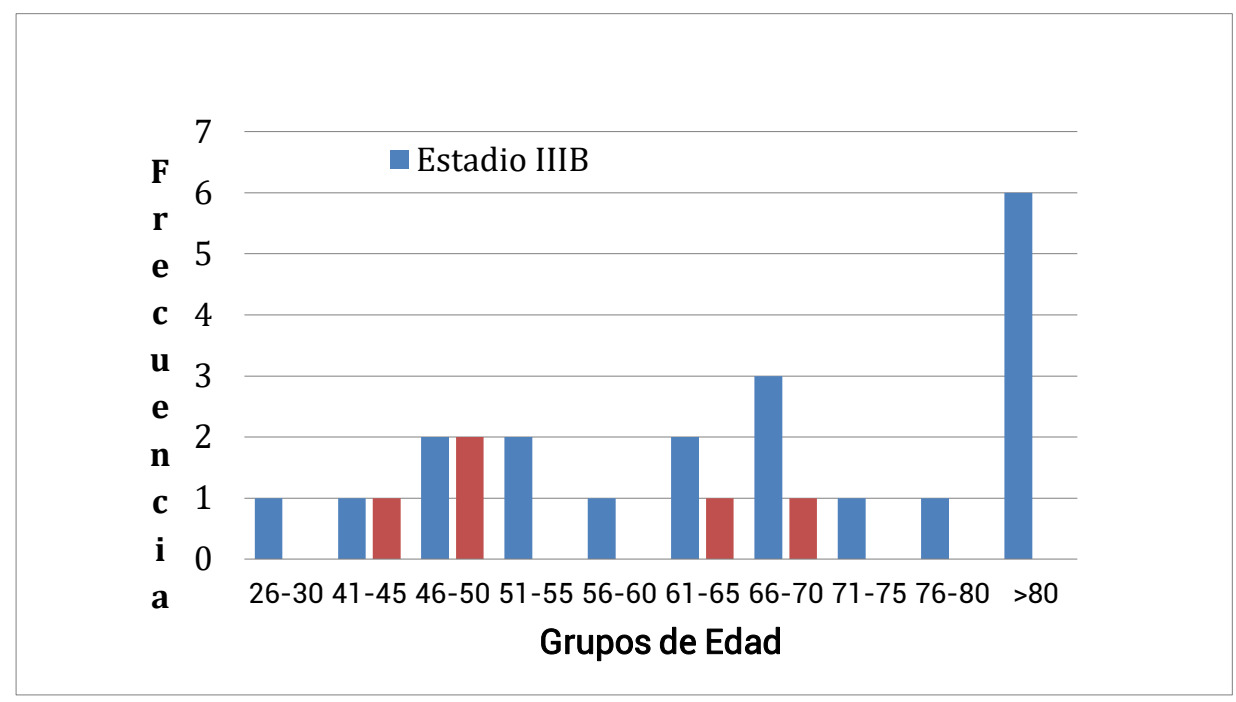

La mediana del tiempo global de tratamiento fue de 65 días, rango de 52 - 80 días. No se observó diferencias al comparar con la mediana del tiempo global de tratamiento de las pacientes que finalmente sobrevivieron más de 5 años: 64.5 días.

Al completar la primera fase de terapia radiante externa (50 Gy), se documentó respuesta parcial en 20 pacientes ( $80 \%$ ), enfermedad estable en las 5 pacientes restantes (20\%); al completar la TRT exclusiva (68 Gy), se encontró respuesta clínicamente completa en 9 pacientes $(36 \%)$, y residual tumoral aparente, clínico, central y parametrial en las 16 pacientes restantes (64\%) (Figura 2). 
Figura 2. Respuesta en primera y segunda fase de radioterapia externa exclusiva.

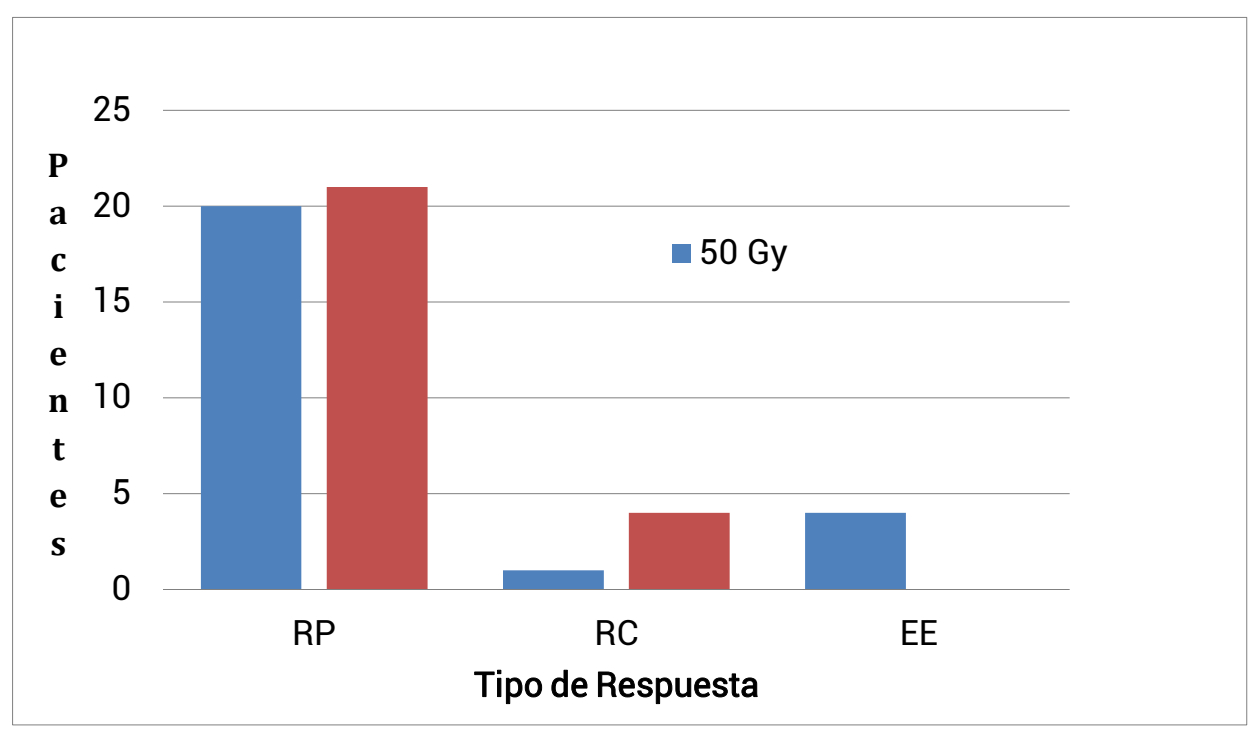

RP: Respuesta parcial, RC: respuesta Completa.

Hubo falla locorregional en 19 pacientes (76\%), en 15 de dichas pacientes, la falla se documentó en los primeros 12 meses, con una mediana de 5 meses. En una paciente se documentó falla distal (unión condrocostal y pulmonar) y no falla locorregional (Figura 3).

A los 5 años de vigilancia, 6 pacientes (24\%) se encontraron libres de enfermedad; 10 pacientes fallecieron con actividad tumoral documentada clínica, citológica o histológica. La mediana de muerte fue a los 11.5 meses; en 7 pacientes no se pudo documentar la fecha de fallecimiento por pérdida del seguimiento, luego del diagnóstico de recidiva tumoral. Finalmente, 2 pacientes, que fueron remitidas a Quimioterapia de rescate, se mantuvieron vivas sin datos de actividad tumoral por más de 5 años. No se obtuvo diferencias estadísticamente significativa en relación al tipo de respuesta obtenida tanto en la primera como en la segunda fase de radioterapia externa exclusiva y la sobrevida global, con un valor P.0.49 (IC al 95\%: 0.2842 - 2.0074) (Figura 4 y 5).

La morbilidad tardía observada fue mayoritariamente grado 2, tanto gastrointestinal baja como urinaria. Una paciente presentó morbilidad gastrointestinal baja grado 3 (Figura 6). 
Figura 3. Falla locoregional por estadíos y tiempos de presentación.

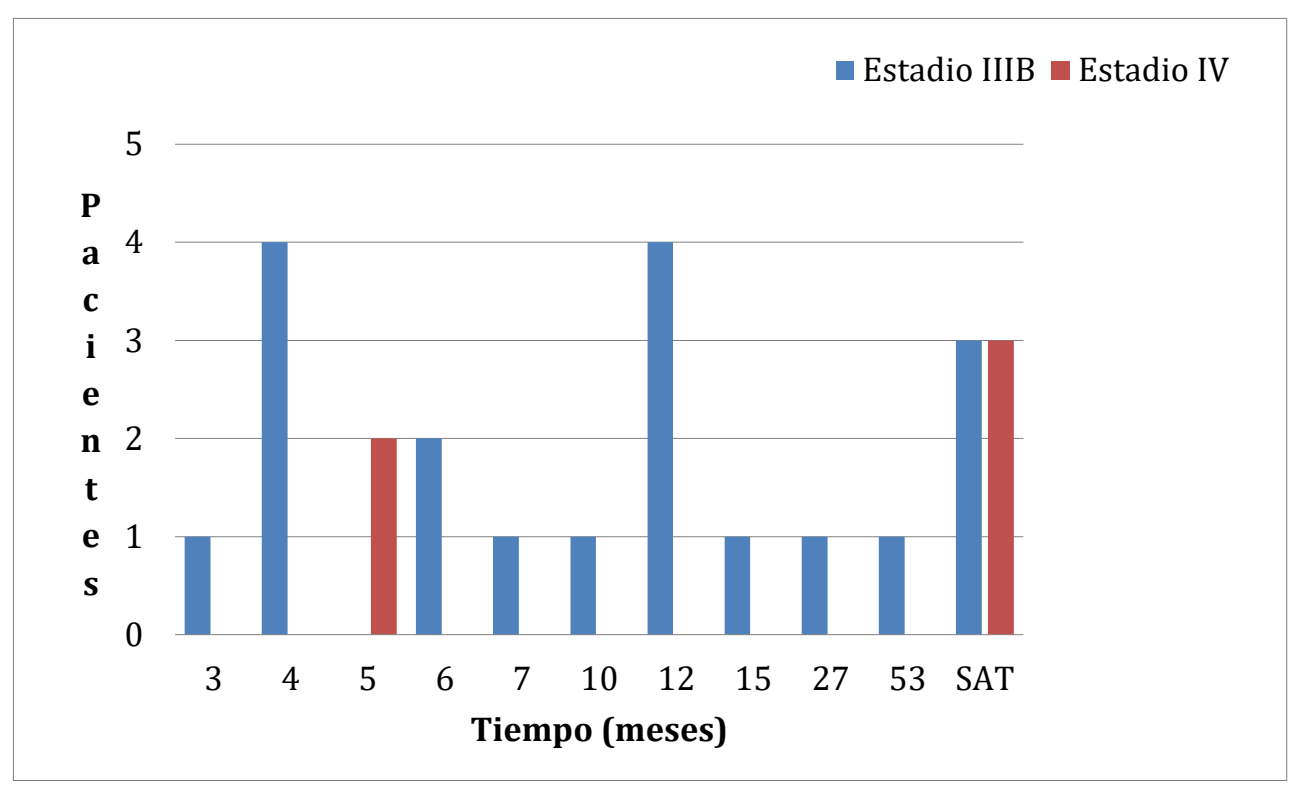

SAT: sin datos de actividad tumoral a 5 años.

Figura 4. Curva de Kaplan Meier supervivencia global

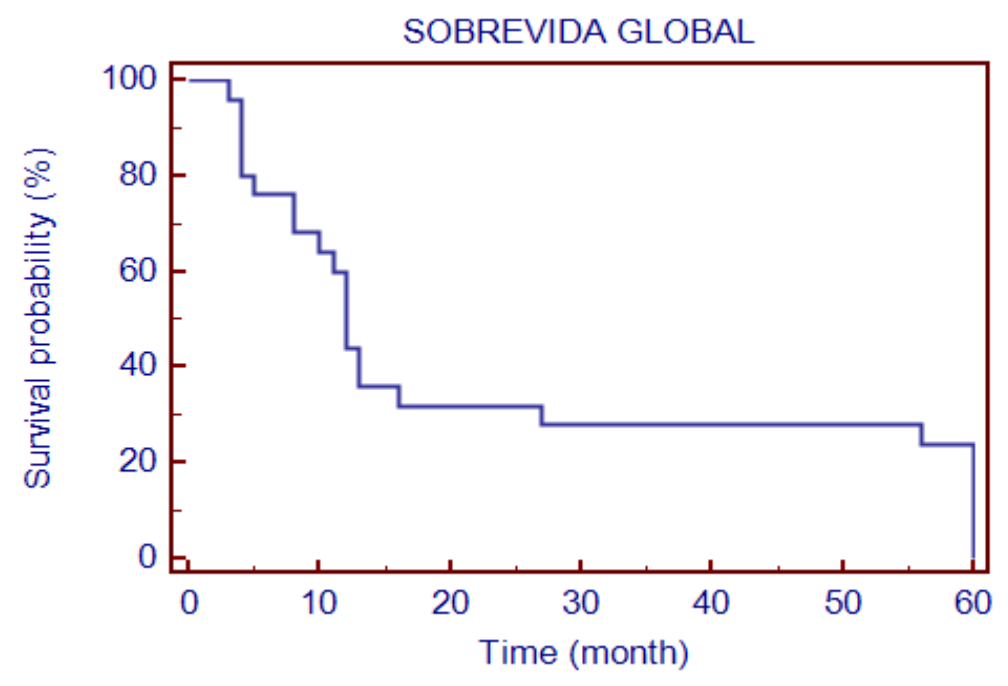


Figura 5. Sobrevida Global por respuesta terapéutica

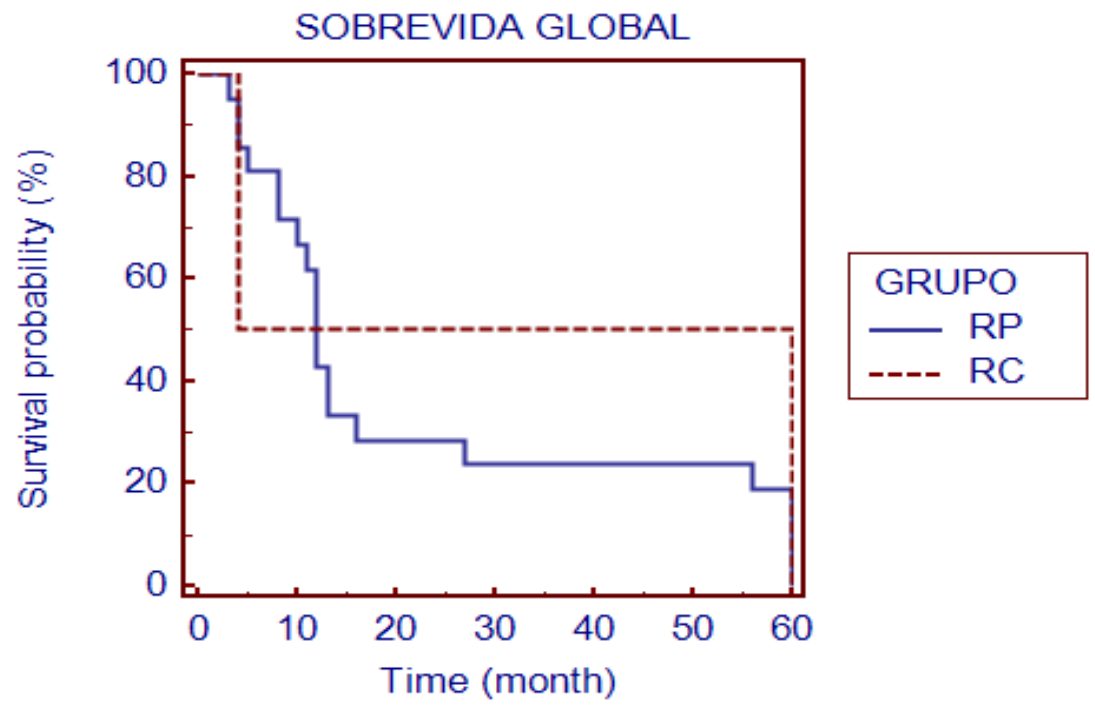

RP: Respuesta Parcial; RC: Respuesta Completa.

Figura 6. Morbilidad tardía en las pacientes con Cáncer de cuello uterino.

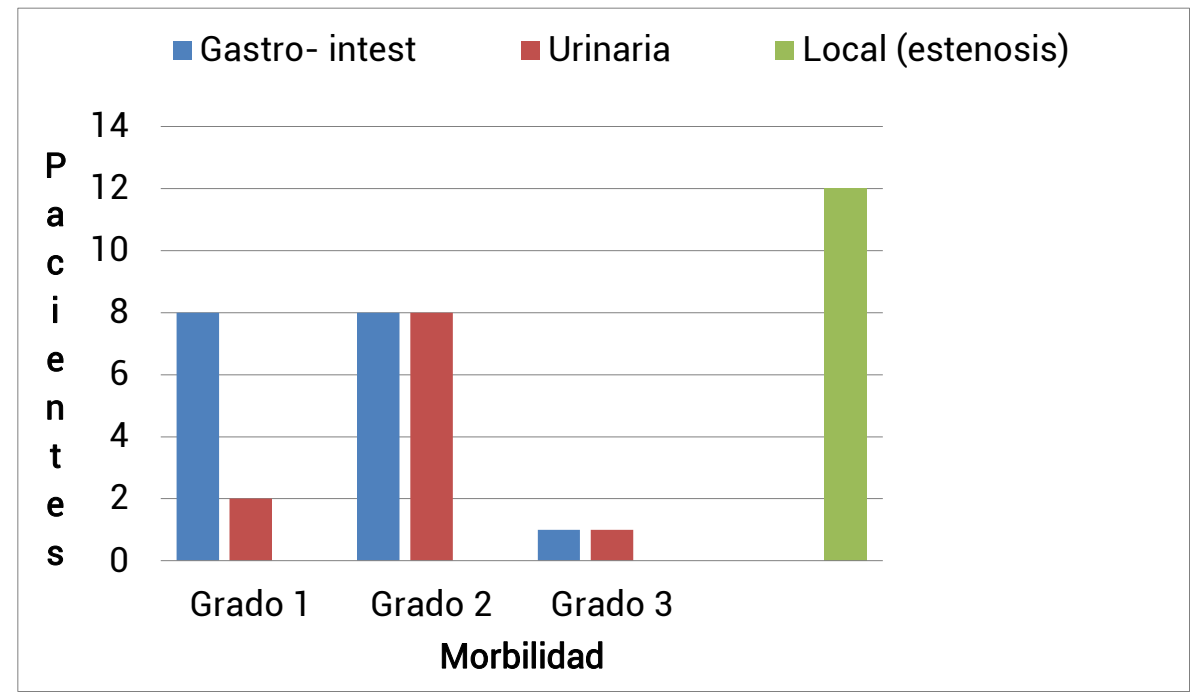

\section{Discusión}

El papel que desempeñan las radiaciones ionizantes en el tratamiento del cáncer invasor del cuello del útero, avanzado, se basa en poder alcanzar dosis altas en el tumor. La BT es decisiva en todos los protocolos de tratamiento. En el Instituto del Cáncer de SOLCA Núcleo de Loja, como en todas las unidades de SOLCA del país, se ha adoptado como guía los Lineamientos NCCN del Instituto del Cáncer de EEUU [10]. Algunos centros oncológicos tienen protocolos que administran BT de entrada [11-13], y/o concomitante a dosis 
moderadas de Radioterapia externa, con resultados similares a los obtenidos con la secuencia de Radioterapia externa - Braquiterapia.

En el presente estudio, se obtuvo una sobrevida libre de enfermedad a 5 años que se observa discretamente mejor a la reportada en los escasos resultados obtenidos en estudios similares, incluso considerando estadios de la enfermedad más tempranos [2-5]. Hanks y colaboradores [14] reporta una falla pélvica de $86 \%$ en pacientes estadio III sometidas a TRT exclusiva, en comparación con la obtenida en los casos reportados del presente estudio en el mismo estadio.

La sobrevida libre de enfermedad a 5 años encontrada en ésta revisión: $24 \%$, en pacientes con estadio clínico III/IV, refleja la importancia de la BT, al compararla con reportes del manejo con TRT + BT, en los mismos estadios: 55 - 64\% [15-17].

La respuesta clínica al término de la primera fase de Teleterapia (50 Gy) tiene implicación pronóstica. Igualmente la respuesta al final de la TRT exclusiva.

La recurrencia locorregional precoz, en los primeros 12 meses de seguimiento, más que recurrencia, hacen pensar en persistencia tumoral, al tener en mente la imposibilidad de alcanzar dosis tumoricidas radicales con TRT exclusiva.

Dada la dosis administrada, la morbilidad tardía observada, predominantemente gastrointestinal y urinaria (grados I y II), es baja y menor a la que reportan los estudios similares comparables, mencionados. Destaca el hecho de encontrar un porcentaje importante de pacientes con estenosis vaginal, secuela observada en las pacientes que, por decisión personal, no han reanudado su vida sexual activa, principalmente por temor e idiosincrasia, pero que, en el seguimiento clínico, impide la visualización o la toma de muestras cito - histológicas para documentar remisión o recurrencia de la actividad tumoral. El presente estudio pretende aportar datos para justificar, a nivel local, la necesidad de contar con equipamiento mínimo para implementar un área de Braquiterapia en nuestro Instituto.

Probablemente, se sigan presentando pacientes que sean candidatas para que reciban TRT exclusiva. Deberá optimizarse la infraestructura con el objeto de poder ofrecer terapia radiante con mejor distribución de dosis (Equipo de Megavoltaje de mayor energía), planificaciones de tratamiento en 3D, IMRT, Radioterapia externa convencional con boost concomitante [4] y, como lo dictan actualmente los lineamientos y protocolos, la asociación de Quimioterapia al esquema de tratamiento.

\section{Conclusiones}

La modalidad de terapia radiante de Radioterapia externa exclusiva para el manejo del cáncer cervical avanzado (estadios III y IVA) ofrece pobres resultados de control y sobrevida, independientemente de la respuesta obtenida tanto en la primera como en la segunda fase de Radioterapia. 


\section{Agradecimientos}

Reconocemos a las personas que participaron indirectamente en el estudio tales como el personal técnico y otras en general del Instituto del Cáncer de SOLCA Núcleo de Loja, a quienes agradecemos.

\section{Información adicional}

Nota del Editor

La Revista Oncología Ecu

permanece neutral con

respecto a los reclamos

jurisdiccionales en mapas publicados y afiliaciones institucionales.
Abreviaturas

BT: Braquiterapia.

RC: respuesta Completa.

RP: Respuesta parcial.

SAT: $\sin$ datos de actividad tumoral a 5 años.

TRT: teleradioterapia.

\section{Archivos Adicionales}

Ninguno declarado por los autores.

Fondos

Los fondos de la investigación fueron propios de los autores del presente artículo.

\section{Disponibilidad de datos y materiales}

Existe la disponibilidad de datos bajo solicitud al autor de correspondencia. No se reportan otros materiales.

\section{Contribuciones de los autores}

LEMV, EAG, ABF realizaron la idea de investigación, revisión bibliográfica, recolección de datos, escritura del artículo. LEMV realizó el análisis crítico del artículo. ABF realizó el análisis estadístico. Todos los autores leyeron y aprobaron la versión final del artículo. 
Aprobación de ética y consentimiento para participar

No aplica ya que es un estudio retrospectivo

\section{Consentimiento para publicación}

No aplica.

\section{Información de los autores}

Luis E Morales Vivar, Instituto del Cáncer de SOLCA Núcleo de Loja, Ecuador.

https://orcid.org/0000-0002-5031-293X

Estrellita Arciniega Gutiérrez, Universidad Nacional de Loja, Ecuador.

Amable Bermeo Flores, Magíster en Salud Pública, Universidad Nacional de Loja.

\section{Revisiones por pares}

Acceda a la revisión de pares académicos en el siguiente enlace: https://publons.com/review/4253492/

\section{Referencias}

Abreviaturas en la referencias

DOI: Digital Object

Identifier

PMID: PubMed Identifier

SU: Short URL
1. Garrido H, Yunga E. Incidencia del Cáncer en Loja, estudio de una década. Registro de Tumores de Loja. Loja; 2010.

2. Ulmer $\mathrm{H}$, Frichbier $\mathrm{H}$. Treatment of advanced cancers of the cervix uterine with irradiation external alone. Int J Radiat Oncol Biol Phys 1983; 9(6):809-812.

3. Akine $Y$, Hashida I, Kajiura $Y$, Watai $K$, Tsukiyama I, Egawa S, et al. Carcinoma of the uterine cervix treated with external irradiation alone. Int J Radiat Oncol Biol Phys 1986;12 (9):1611-1616.

4. Matsuura $\mathrm{K}$, Okabel $\mathrm{T}$, Fujita $\mathrm{K}$, Tanimoto $\mathrm{H}$, Akagi $\mathrm{Y}$, Kgemoto $\mathrm{M}$. Clinical results of external beam radiotherapy alone with a concomitant boost program or with conventional fractionation for cervical cancer patients who did not receive intracavitary brachytherapy. Radiat Res 2012;53(6):900-5. DOI: 10.1093/jrr/rrs051.

5. Castro JR, Issa P, Fletcher GH. Carcinoma of the cervix treated by external irradiation alone. Radiology 1970;95(1):163-6.

6. Coia L, Won M, Lanciano R, Marcial VA, Martz K, Hanks G. The patterns of care outcome study for cancer of the uterine cervix: Results of the Second National Practice Survey. Cancer. 1990;66(12):2451-6.

7. Montana GS, Fowler WC, Varia MA, Walton LA, Mack Y, Shemanski L, Montana C, et al. Carcinoma of the cervix, stage III: Results of radiation therapy. Cancer 1986;57:148-154. 
8. Saibishkumar EP, Patel FD, Sharma SC, Karunanidhi G, Sankar AS, Mallick I. Results of external-beam radiotherapy alone in invasive cancer of the uterine cervix: a retrospective analysis. Clin Oncol ( $\mathrm{R}$ Coll Radiol) 2006;18(1):46-51.

9. Cox TD, Stetz J, Pejak TF. Toxicity criteria of the Radiation Therapy Oncology Group (RTOG) and the European Organization for Research and Treatment of Cancer (EORTC). Int $\mathrm{J}$ Radiat Oncol Biol Phys 1995;31(5):1341-6.

10. National Comprehensive Cancer Network. NCCN Clinical Practice Guidelines in Oncology (NCCN Guidelines): Cervical cancer. Version 2.2013.

11. Ota T, Takeshima N, Tabata T, Hasumi K, Takizawa K. Treatment of squamous cell carcinoma of the uterine cervix with radiation therapy alone: long-term survival, late complications, and incidence of second cancers. British Journal of Cancer 2007;57(1):148-54.

12. Rose PG, Bundy BN, Watkins ET, Thigpen T, Deppe G, Maiman MA, et al. Concurrent cisplatin-based radiotherapy and chemotherapy for locally advanced cervical cancer. N Eng J Med 1999;340(15):114453.

13. Whitney CW, Sause W, Bundy BN. Randomized comparison of fluorouracil plus cisplatin vs. hydroxyurea as an adjunct to radiation therapy in stage IIB-IVA carcinoma of the cervix with negative paraaortic lymph nodes: A Gynecologic Oncology Group and Southwest Oncology Group study. J Clin Oncol 1999;17(5):1339-48.

14. 14. Hanks C. Patterns of care outcome studies: Results of the national practice in cancer of the cervix. Cancer 1983;51: 959-967.

15. Gaffney D, Du Bois A, Narayan K, Reed N, Toita T, Pignata S, et al. Practice patterns of radiotherapy in cervical cancer among members groups of the Gynecologic Cancer Intergroup (GCIG). Int J Radiat Oncol Biol Phys 2007:68(2): 485-90.

16. Jones B, Tan LT, Blake PR, Dale RG. Results of a questionnaire regarding the practice of radiotherapy for carcinoma of the cervix in the UK. Br J Radiol 1994;67:1226-1230.

17. Lanciano RM, Pajak TF, Martz K, Hanks GE. The influence of treatment time on outcome for squamous cell cancer of the uterine cervix treated with radiation: A patterns-of-care study. Int $\mathrm{J}$ Radiat Biol Phys 1993;25(3):391-7. 